\title{
Modern measurement methods in strength tests of sigma thin-walled steel beams
}

\author{
Ilona Szewczak ${ }^{1, *}$, Katarzyna Rzeszut ${ }^{2}$ \\ ${ }^{1}$ Lublin University of Technology, Faculty of Civil Engineering and Architecture, ul.Nadbystrzycka 38d, \\ 20-618 Lublin, \\ ${ }^{2}$ Poznan University of Technology, Institute of Structural Engineering, Marii Skłodowskiej-Curie 5, \\ 60-965 Poznań, Poland
}

\begin{abstract}
In the paper the modern measurement methods of structural deformation using GOM optical measuring systems based on digital image processing were presented. First part of the work demonstrates the results of measurement of initial geometrical imperfections of steel structural elements using the Tritop optical 3D coordinate measuring machine. The method of preparation of sigma type steel beams with a height of $200 \mathrm{~mm}$, a flange width of $70 \mathrm{~mm}$ and a wall thickness of $2 \mathrm{~mm}$ is presented. Afterwards, the subsequent stages of measurements and the method of reading the obtained results and their interpretation are described. The next step presents a test stand designed to perform strength tests of thin-walled sigma beams in a four-point bending scheme. In addition, the method of preparation of beam's surface to measurement of $3 \mathrm{D}$ using the Aramis system and electrofusion strain gauges is presented. The deformation values obtained for three sigma-type steel beams were compared with respect of two mentioned measurement methods. The final conclusions and recommendations regarding the methodology of geometry and strain measurement using optical measuring systems complete the work.
\end{abstract}

\section{Introduction}

Laboratory tests of thin-walled cold-formed steel elements as well as hot-rolled elements with one axis of symmetry have caused many problems for scientists over the years. During the bending laboratory tests, the non-symmetrical beams are subject also to significant torsion, which results from the fact that the load is usually applied to the element's flange. Then the resultant force, which is located in the middle of the width of the flange, causes additional torsion due to eccentricity of the shear centre. In such cases, a particularly difficult issue is the correct measurement of displacements of steel structural elements, because the classic method of measuring displacements using LVDT inductive sensors fails. The authors of this article were able to experience this in person during the pilot tests of sigma thin-walled steel beams under uniformly distributed loads presented in paper [1]. Due to the significant torsion of the tested beams and large displacements out of the plane,

\footnotetext{
*Corresponding author: i.szewczak@pollub.pl
} 
the readings from the inductive sensors were incorrect. In fact, the detector's head of inductive sensor slid off the original measuring point and recorded the displacement in a different point. Therefore, to avoid this problem, the use of modern measurement methods such as optical measuring systems based on digital image processing is proposed in this paper.

Software for digital image correlation is increasingly used and described in literature. For example, in research on intensity factor for cracked steel I-beams under both bending moment and axial load, a 3D digital image correlation system was used [2]. By means of this measurement authors illustrate the stress evolution pattern and the plasticity zone around the crack tip and used it for further verification of the theoretical models. In the next paper work regarding the behaviour of notched steel beams reinforced by non-prestressed and pre-stressed bonded CFRP, an optical image correlation system was used. The measurements provide information about stress distribution at the vicinity of the crack tip [3]. In the literature, one can also find examples that image correlation is a new technology that can be used to evaluate the structural response of reinforced FRP structures. For example in [4], a 2D image correlation measurement system was used for the assessment of the strains in pull-off tests conducted on FRP composites glued to concrete blocks.

In [5], authors have discussed the possibilities of using the non-destructive testing methods to characterize the polymeric composites and proved that the Aramis system is a useful tool in three-dimensional strain measurement on the sample surface during the tensile test.

\section{Preparation of test samples}

Before testing with the Aramis 3D sensor and Tritop measuring machine, the sample must be specially prepared. Due to the fact that both systems use optical measurements, the surface of the tested steel beams cannot cause reflection.

Before performing the laboratory tests using Aramis system, all steel beams were degreased and painted with matt white colour spray paint. Then the surface was additionally tarnished by spraying white chalk on it. After drying the previously painted surface, the texture was made in the form of black irregular speckle patterns. It was prepared by using a black matte oil paint applied using a special sponge. In this way, the inner and outer surface of the cross-sectional walls of the beams was prepared on more than half of their length. Such preparation of the material for research is undoubtedly timeconsuming. The scope of the Aramis lens test included an area of length $140 \mathrm{~cm}$, which is almost half of the span of the tested beams.

In order to verify the measurements of deformations made by the Aramis system, electrofusion strain gauges TENMEX TFs-10 with resistance of $120 \Omega \pm 0.2 \%$ were bonded in the middle of the beam span. The electrofusion strain gauges (ESG) were placed on the not painted side of walls. The location of ESG is shown in Figure 1a.

In order to verify the measurements of deformations made by the Aramis system, electrofusion strain gauges TENMEX TFs-10 with a resistance of $120 \pm 0.2 \%$ were adhered at mid span of the beam. Electrofusion strain gauges (ESG) were placed on the unpainted side of the walls. The location of the ESG is shown in Figure 1a. Figure 1a also shows measuring points with a diameter of $5 \mathrm{~mm}$, enabling the initial measurements of geometrical imperfections using the Tritop system, which were later used to make a common coordinate system for two measuring lenses in the Aramis system. In these points, it is also possible to analyze the displacements of the tested beams in the GOM Correlate program. Beams completely prepared for testing are shown in Figure $1 \mathrm{~b}$. 

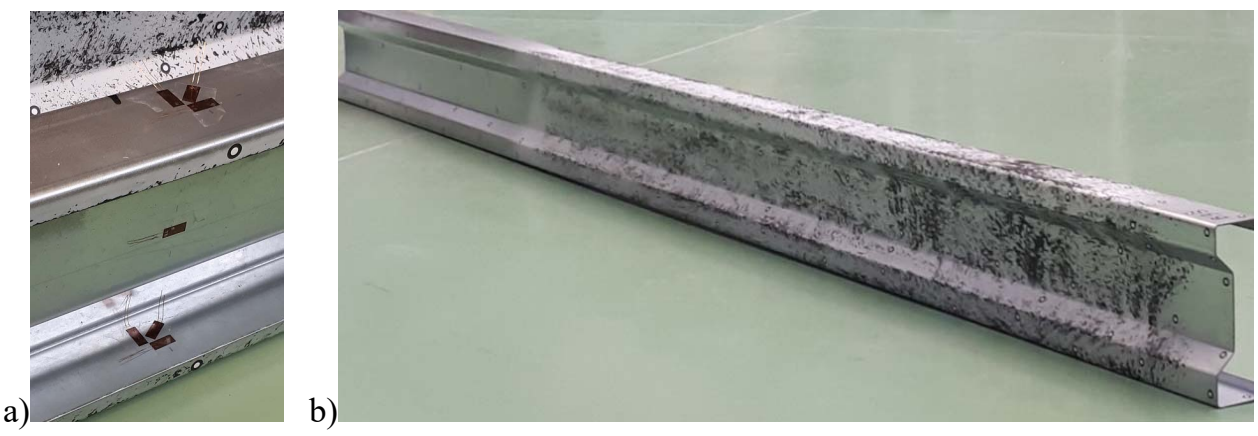

Fig. 1. Preparation of test samples a) location of ESG; b) beams completely prepared for testing.

\section{Measurement of the initial beam geometry using TRITOP}

Optical 3D coordinate measuring machine - Tritop, is a portable system enabling precise and quick measurements of coordinates of three-dimensional objects. It does not require any complex, heavy and maintenance-intensive hardware. It consists of a camera, which photos of the object are directly sent to the computer with GOM Correlate software that allows controlling the correctness of the taken pictures, based on coded points and two invar alloy length patterns. Inwar is an alloy with a constant coefficient of thermal expansion. The standard length pattern is certified at $20^{\circ} \mathrm{C}$. During the tests, two length patterns are placed next to the analysed object - one allows to control the other, and the system gives precisely the ambient temperature, which allows for a very accurate measurement.

The measurement layout is shown in Fig. 2. The test is simple and it is based on taking a series of correct photos. Tritop catches a white measuring point placed on tested element and also coded points used to create a coordinate system. If the photo does not contain the appropriate number of measuring and coded points, the system informs about it with a sound signal. When the photos are made properly, all points should be marked in green, as shown in Fig. 2. Tritop operates on the principle of short-range photogrammetry. On the basis of a series of photos, the system reads the angle of the test from two sides of the element and enables the reproduction of the external orientation of the images based on the arrangement of coded points and the pattern. Thus, it allows precise measurements of individual dimensions of the element in the $3 \mathrm{D}$ coordinate system and the measurement of angles between the planes, as shown in Fig. 3.

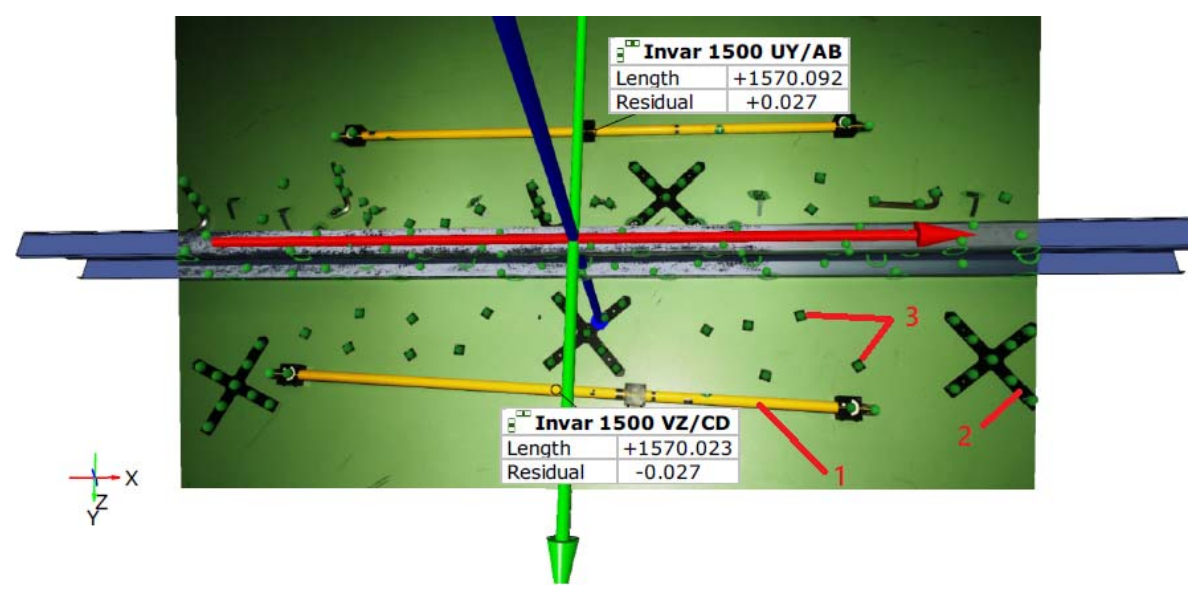

Fig. 2. Measurement of geometrical imperfections of beams using TRITOP. 1 - invar length pattern, 2 - large coded points, 3 - small coded points. 
a)

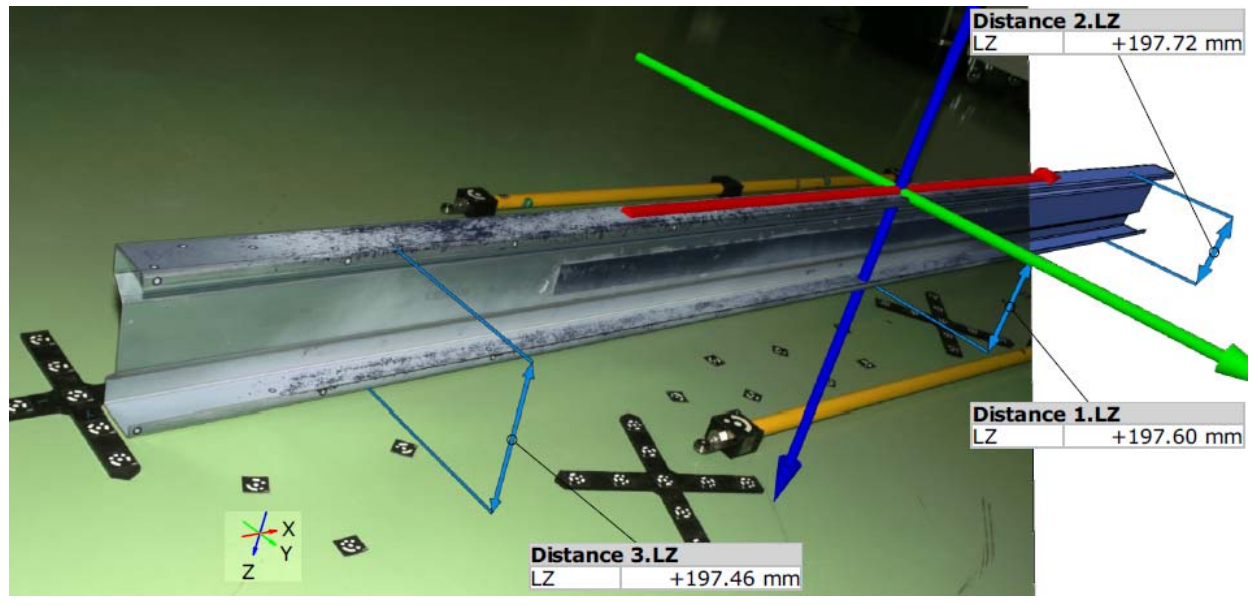

b)

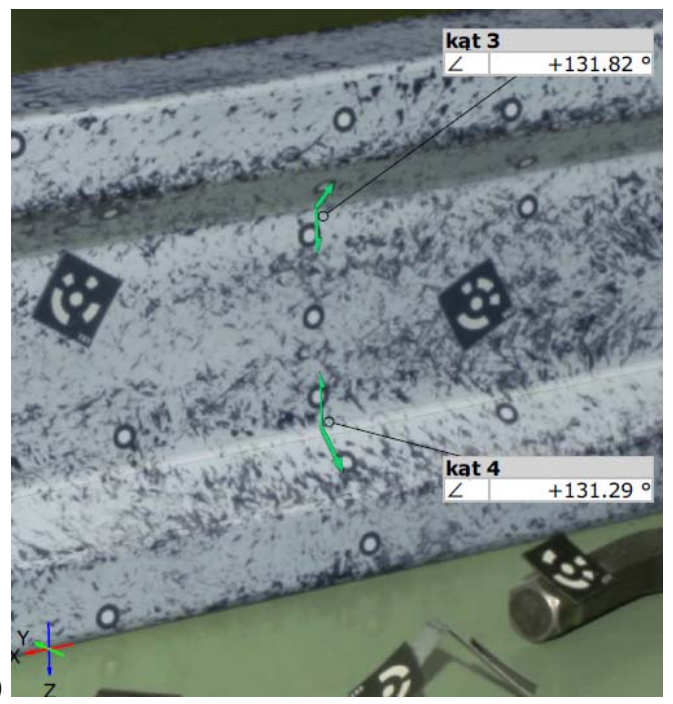

Fig. 3. Geometry measurements using the Tritop machine, measurement of: a) height, b) angles between planes.

\section{Measurement of beam displacements and strain in the Aramis system}

Aramis is a non-contact measuring system based on digital image correlation (DIC). It allows for very precise measurements of $3 \mathrm{D}$ coordinates, deformations or displacements, with accuracy to sub micrometres, regardless of the geometrical characteristics of the object and its temperature. It also provides very high accuracy of measurements on samples tested under static and dynamic load. The principle of the system is based on stereo photogrammetry. One system consists of two cameras, set at a known angle and at a certain distance. Before each measurement, the system is calibrated using a measuring cross.

In this study two Aramis systems was used in laboratory tests of sigma thin-walled steel beams. This was due to the fact that the sigma section was mono-symmetrical and the authors were interested in the measurement of deformations and displacements from both sides of the element. In order to ensure cooperation between the two measurement systems, 
a common coordinate system was created, for which the Aramis system used measuring points located on samples for prior tests with the Tritop machine (Fig. 4).

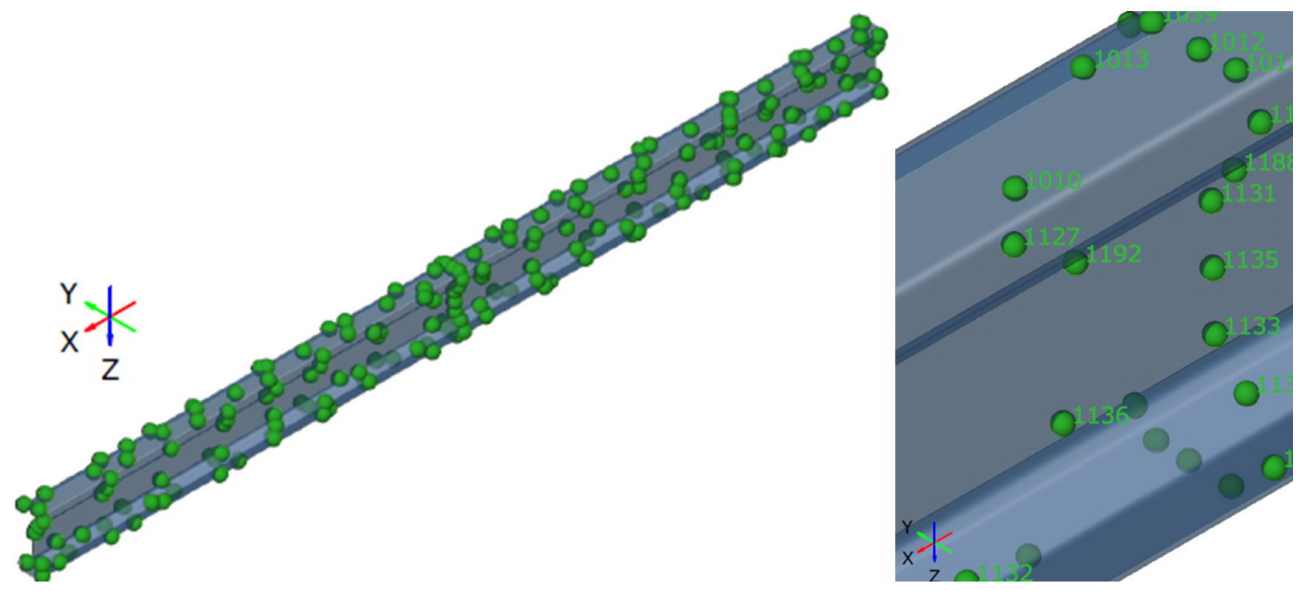

Fig. 4. Layout of points used to create a coordinate system in the Aramis system.

The analysis of the obtained measurements performed in the GOM Correlate program allows for the image shown in Fig. 5, in which the image of two cameras is oriented in space. In addition, it is possible to synchronize readings from the Aramis system with the load increment on the press of a testing machine. The accuracy of the obtained results depends on the number of photos taken by the system during the test.

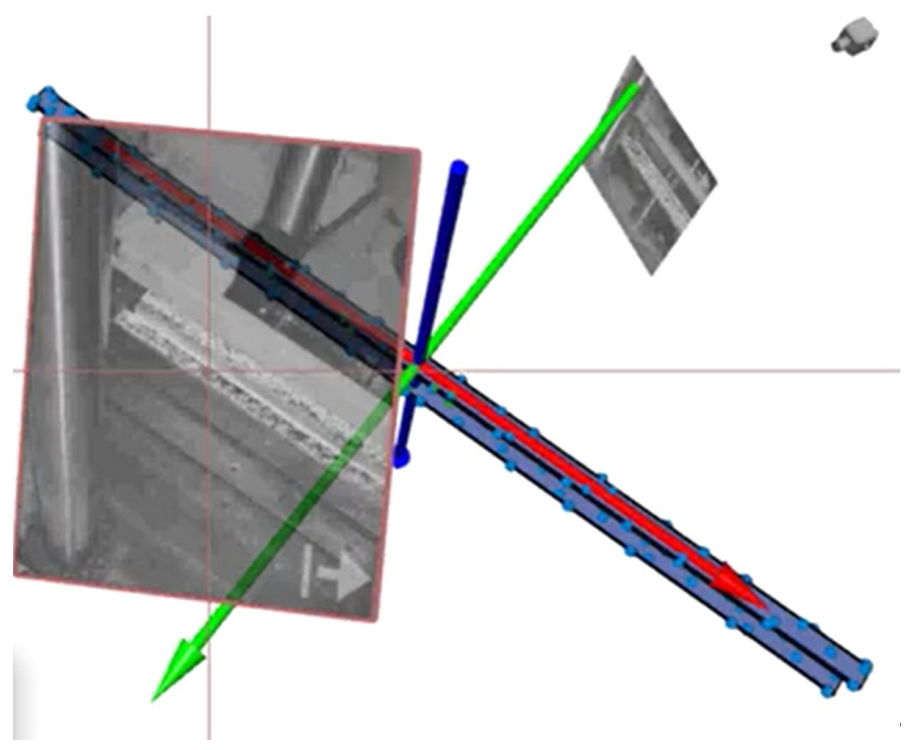

Fig. 5. The image of two Aramis cameras processed with GOM Correlate.

The system allows the presentation of the results in the form of video, charts and presentations. Examples of the photo film presenting movement of a selected point on the beam in the 3D space are shown in Fig. 6. 


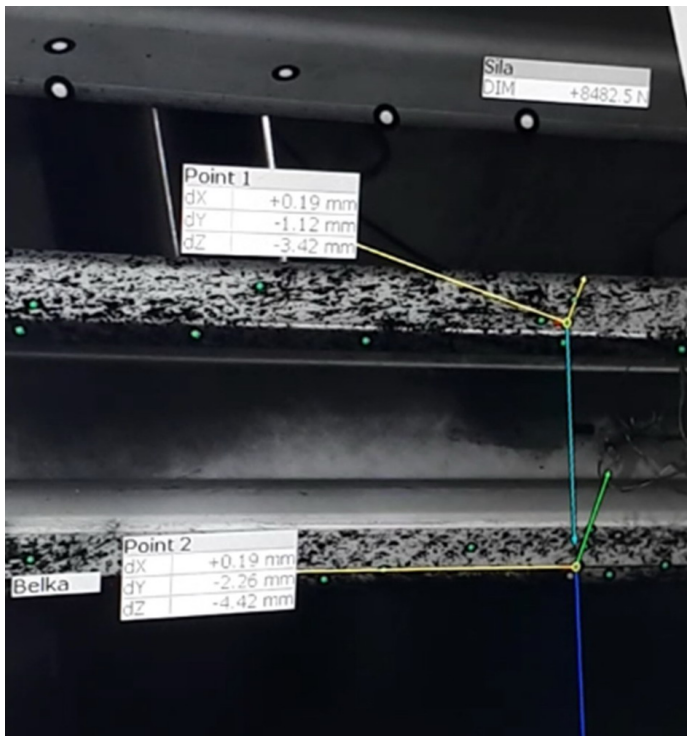

Fig. 6. Representation of displacement measurement in the GOM Correlate.

\section{Laboratory test stand}

Three thin-walled steel beams of "Blachy Pruszynski" $\Sigma 200 \times 70 \times 2$ cross-section with span $3 \mathrm{~m}$ and grade of steel S350GD were tested. The beams were examined in a four-point bending scheme. The spacing of supports was $270 \mathrm{~cm}$ and the distance between two concentrated loads was $135 \mathrm{~cm}$. The supports used in the experiment mapped theoretical fork support. The test stand is shown in Fig. 7.
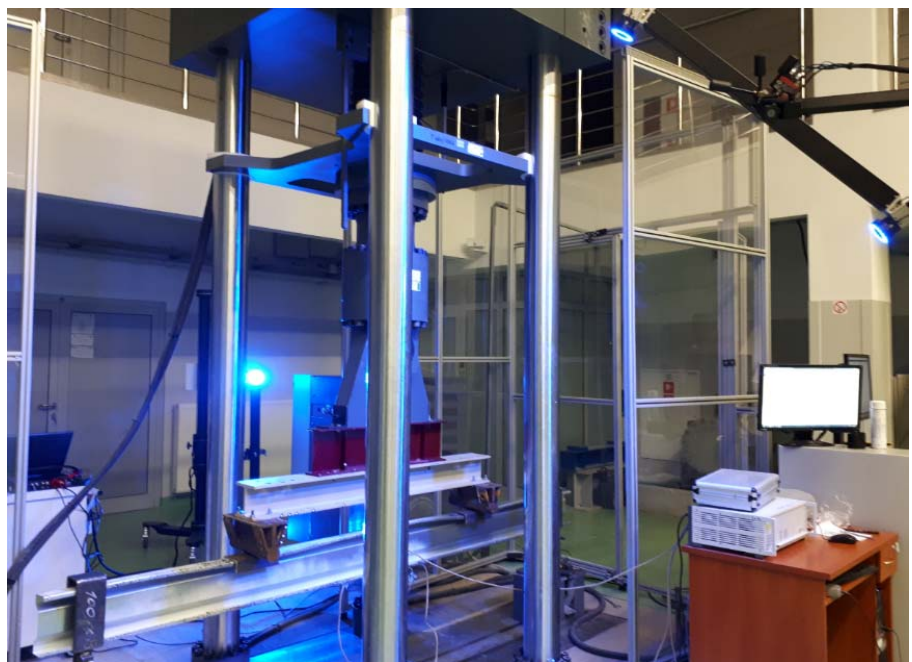

Fig. 7. Laboratory test stand with two Aramis cameras.

During the laboratory test, displacements and deformations of the beam were measured using the Aramis system, and strains were controlled using electrofusion strain gauges placed in the middle of the beam span in the upper, lower flange and in the web. 


\section{Analysis of the obtained results}

Laboratory tests were preceded by the measurement of initial geometric imperfections of steel beams. The obtained results are presented in Fig. 8a. Using GOM Correlate software the image received from measurements can be adapted to a previously created beam model in accordance with the manufacturer's data. This allows the creation of a 3D model, which later can be transfer to programs for structural analysis, eg Abaqus, to perform a detailed numerical analysis of a given beam. Thus, greater consistency between the results of laboratory and numerical tests can be achieved. It should be noted that in the case of thinwalled beams, any initial geometrical imperfections can have a significant impact on displacement and strain magnitude.

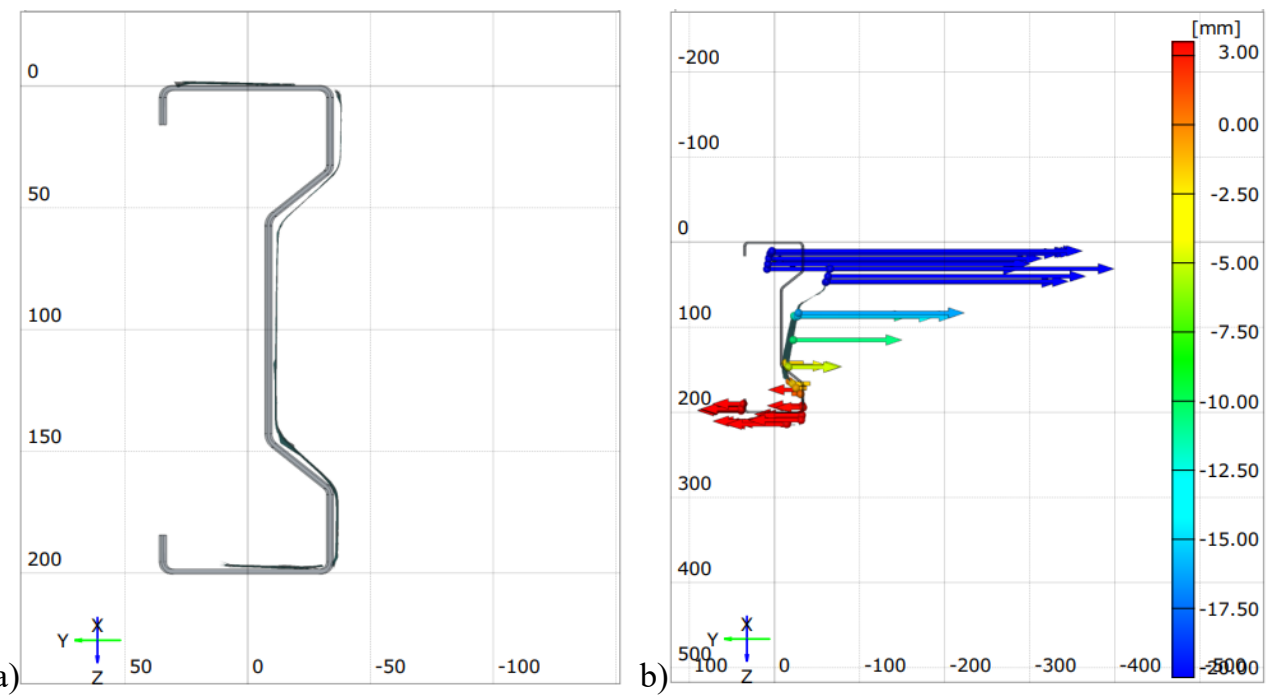

Fig. 8. Result of measurement a) initial geometrical imperfections of the beam, b) beam displacement obtained from Tritop and Aramis system.
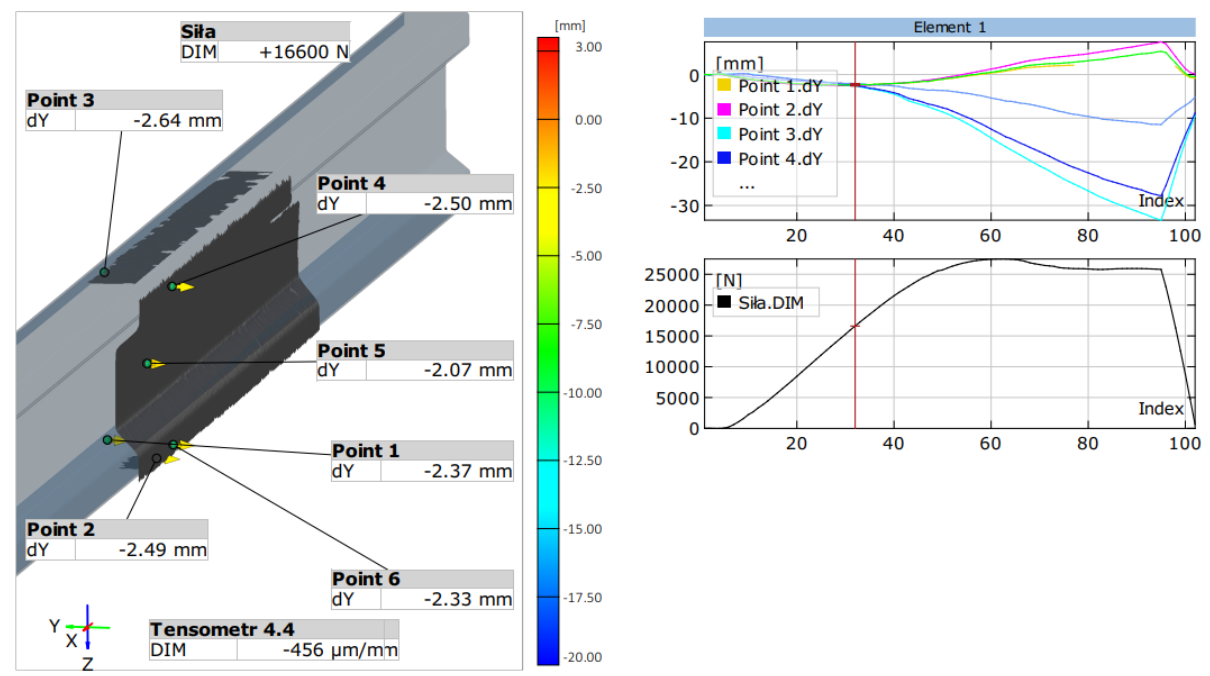

Fig. 9. Displacements with respect to the $\mathrm{Y}$ axis at several points in the middle of the beam span. 
Points located on a steel beam in order to perform measurements using Tritop machine can also be used for the purposes of Aramis system during the strength tests. The obtained results can be presented in the form shown in Figure 8b, or in the form of a 3D image with graphs, as shown in Figure 9.

During the test, beam strains were also measured using electrofusion strain gauges and the Aramis system. The obtained results for two selected electrofusion strain gauges are listed in Fig. 10 and 11. The Aramis system allows analysing deformations in any direction, where the measurement is performed in a certain area. The measurement is based on determining the deformations between the black speckles of black and white speckle patterns. Figure 10 shows the relation load-strain obtained using electrofusion strain gauge placed on the inner plane of the upper flange (compressed) in the middle of the beam span in the longitudinal direction of the beam (T2-T) and measured in the same area on the outer plane of the upper flange using Aramis system (T2-A).

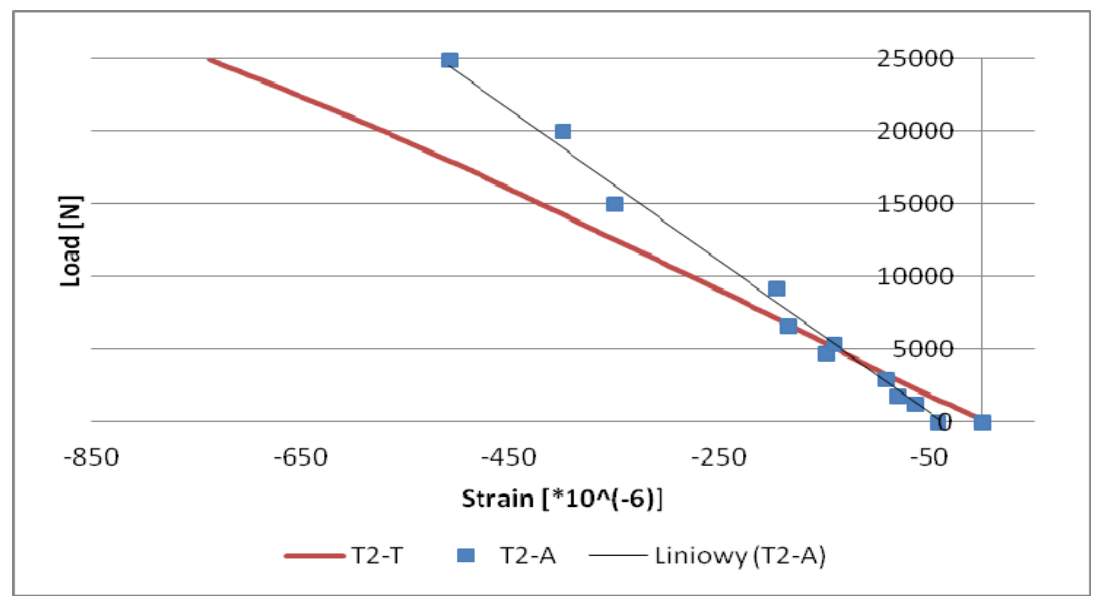

Fig. 10. Load-strain relation at the upper flange using a electrofusion strain gauge (T2-T) and Aramis system (T2-A, Liniowy (T2-A)).

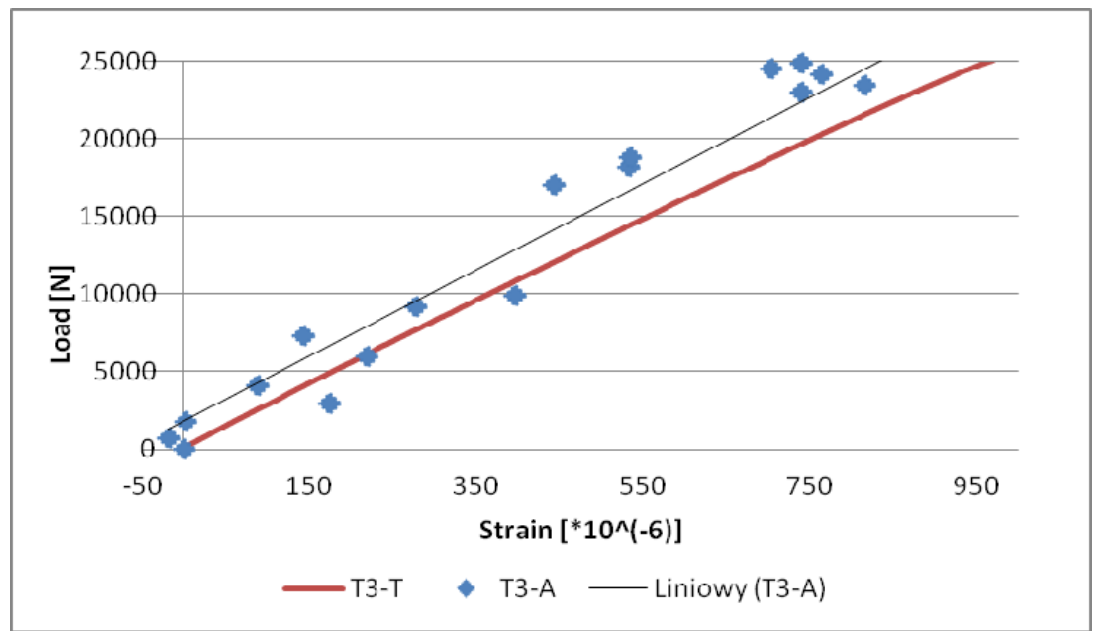

Fig. 11. Load-strain relation at the bottom flange using a electrofusion strain gauge (T3-T) and Aramis system (T3-A, Liniowy (T3-A)). 
Figure 11 shows the relation load-strain obtained using electrofusion strain gauge placed on the outer plane of the bottom flange (tensioned) in the middle of the beam span in the longitudinal direction of the beam (T3-T) and measured in the same area on the inner plane of the bottom flange using the Aramis system (T3-A).

The results obtained with the use of the Aramis system (T3-A - marked with points) show significant noise, but the trend line (Liniowy T3-A) is similar to the readings obtained from the electrofusion strain gauge. Observed differences may result from the fact that the strain may slightly differ through the wall thickness of the beam $(2 \mathrm{~mm})$. In addition, the strain gauges may not have been located perfectly according the symmetry axis of the beam flange. Moreover measurements from the Aramis system are read from a slightly larger field than in case of strain gauge. During the tests, the Aramis system was used to analyse the entire steel beam. Thus, two measuring lenses were used that were set at a quite large distance and at a high angle to allow analysis of a $1.4 \mathrm{~m}$ wide field. In order to compare the strains magnitude from electrolytic strain gauge with the Aramis system, a small field (approx. $\mathrm{cm}^{2}$ ) was numerically cut in the GOM Correlate program near the strain gauge. A digital image from such a small field is characterized by a small number of pixels, so the system reads the deformation with no more than $1 \%$ of the points of the whole image, which affects the poor accuracy of the analysis. This observation was also widely discussed in [6]. Note that the main purpose of using the Aramis system for research presented in this paper was to precisely measure displacements, which was achieved. However, in order to measure strains, the Aramis system should be focused on a small area.

\section{Conclusions}

The paper presents new techniques for measuring displacements and deformations of elements subjected to strength tests.

When measuring displacements of steel thin-walled beams, the TRITOP and Aramis systems allow for very accurate measurements in 3D space and software GOM Correlate allows spatial representation of results. Therefore, it is possible to obtain displacement readings in each direction, which allows for a full analysis and a better comparison of results obtained from laboratory tests and numerical analyses, which additionally allows for exact verification and validation of numerical models. The preparation of samples for testing is admittedly quite time-consuming, due to the necessity of sticking a significant number of measuring points or applying the appropriate pattern of patches, but gives much larger measurement possibilities compared to traditional inductive sensors, especially in the case of non-symmetric cross-sections. It was proved by authors in the pilot tests, when inductive sensors did not allow investigating displacements at a specific point, because the sigma beam was subjected to the significant torsion and the sensor slid off the measuring point.

Next part of the study pertains to the measurement of strains, which was carried out using the Aramis system and traditional electrofusion strain gauges. It has been found that the preparation of the desired patches pattern on the beams surfaces required by Aramis is a time-consuming work but comparable to the time of sticking the electrofusion strain gauges to the beam. It is worth noting that the Aramis system allows reading the strain in all directions, while the reading from the strain gauges depends on their orientation. Electrofusion strain gauges are disposable and quite expensive, which limits the number of measurement points. Whereas the cost of using the Aramis system is related to the cost of buying or renting it, but allows for the collection of deformations in an unlimited number of surfaces and in any direction.

In order to use the measurement capabilities of the system properly, one should answer the question for what purpose the system should be used and then properly select the size of 
the area to be examined and, consequently, the type of the measuring lens. The quality of the obtained results is also influenced by the selection of the speed of taking measurements, the quality of the pattern patches and the angle of the lens. The inevitable selection of these parameters may cause significant "noise" in the results. When testing a small surface area differences between the readings of a strain gauge and the Aramis system should not exceed about $2-3 \%$.

Summing up, the use of modern measurement systems in laboratory research opens up new possibilities for scientists in the area of a more comprehensive analysis of the structural behaviour. The use of optical measurement systems based on digital image processing enables attractive presentation of results, allows advanced analysis of results and the possibility of full comparison with numerical analyses. However, they do not replace traditional measurement methods, but only expand and complement them. However, it is always worth reaching for proven, traditional measurement methods to allow control over the obtained results.

Acknowledgments. Financial support by the "Młoda Kadra" Lublin University of Technology, Financial support by the DS grant 01/11/DSPB/0006 Poznan University of Technology, company „Blachy Pruszyński” and „SIKA” is kindly acknowledged.

\section{References}

1. K. Rzeszut, I. Szewczak, International Journal of Civil, Environmental, Structural, Construction and Architectural Engineering, Vol:11, No:7 (2017)

2. E. Ghafoori, M. Motavalli, Engineering Fracture Mechanics 78, 3226-3242 (2011)

3. E. Ghafoori, A. Schumacher, M. Motavalli, Fatigue behavior of notched steel beams reinforced with bonded CFRP plates, Engineerig Structures 45, 270-283 (2012)

4. M. Ali-Ahmad, K. Subramaniam, M. Ghosn, J Eng Mech, ASCE 132(9), 914-23 (2006)

5. R. Oliwa, Mechanik 12/2015, 147-151 (2015)

6. P. Mazzoleni, Uncertainty estimation and reduction in digital image correlation measurements, Doctoral Dissertation Politecnico di Milano, (2013) 